Int. J. Dev. Biol. 58: 705-711 (2014)

doi: 10.1387/ijdb.150032yi

\title{
Characterization of the insulin-like growth factor binding protein family in Xenopus tropicalis
}

\author{
YOSHIKAZU HARAMOTO ${ }^{1}$, TOMOMI OSHIMA ${ }^{1, \#, ~ S H U J I ~ T A K A H A S H I ~}{ }^{2}$ and YUZURU ITO*,1 \\ ${ }^{1}$ Research Center for Stem Cell Engineering, National Institute of Advanced Industrial Science and Technology \\ (AIST), Tsukuba, Ibaraki, Japan and Institute for Amphibian Biology, Graduate School of Science, \\ Hiroshima University, Higashi-Hiroshima, Hiroshima, Japan
}

\begin{abstract}
The insulin-like growth factor binding protein (Igfbp) family consists of six members designated lgfbp1-6. Igfbps are involved in many vital biological functions. They physically interact with IGFs (IGF1 and IGF2) and act as carriers, thereby protecting IGFs from proteolytic degradation. Thus, they function as modulators of IGF activity. Furthermore, Igfbps have been reported to have IGF-independent activities. They interact with other proteins, including cell surface proteins, extracellular matrix proteins, and potentially intracellular molecules. In Xenopus tropicalis (X. tropicalis), only four igfbp genes (igfbp1, igfbp2, igfbp4, and igfbp5) have been identified, and their expression is not well characterized. We report that $X$. tropicalis genome lacks the igfbp 3 and igfbp 6 genes based on synteny analyses. We also examined the spatio-temporal expression patterns of igfbp genes in early $X$. tropicalis development. Expression analyses indicated that they are differentially expressed during early development. Each igfbp gene showed a characteristic spatial expression pattern. Except for igfbp5, they demonstrated overlapping expression in the pronephros. The Xenopus pronephros is composed of four domains (i.e., the proximal tubule, intermediate tubule, distal tubule, and connecting tubule). Our results showed that at least two igfbp genes are co-expressed in all pronephric domains, suggesting that redundant functions of igfbp genes are required in early pronephric kidney development.
\end{abstract}

KEY WORDS: insulin-like growth factor (IGF), insulin-like growth factor binding protein (IGFBP), Xenopus tropicalis

Insulin-like growth factors (IGF1 and IGF2) are important in regulating cellular growth and differentiation. Their functions are mediated by the IGF-1 receptor and IGF-2 receptor (mannose-6-phosphate receptor) (reviewed in Nakae et al., 2001). The functions of IGFs are modulated by a family of binding proteins termed insulin-like growth factor binding proteins (lgfbps). Igfbps include six members designated Igfbp1 through Igfbp6, and are grouped based on conservation of gene organization, structural similarity, and IGF binding affinity. Igfbps are unusually multifaceted molecules. They distribute IGFs and modulate IGF binding to receptors; therefore, they play a significant role in mediating IGF actions. In addition to their role as IGF carriers, they also regulate IGFs either by inhibiting their binding to receptors or potentiating activities, protecting IGFs from protein degradation (reviewed in Hwa et al., 1999).

Igfbps can also function in IGF-independent manners. They interact with the extracellular matrix and cell surface proteins including integrins. Igfbps are transported into the nucleus via nuclear localization signaling and exert IGF-independent effects by transcriptional modulation of genes (reviewed in Hwa et al., 1999). Understanding the functions of Igfbps in vivo has been difficult, largely because lgfbp knockout mice have no dramatic phenotypes. Examinations of the multiple functions of these proteins and redundancy in their expression in various tissue types will be necessary.

In Xenopus laevis, the expression patterns of only two igfbp genes, igfbp4 and igfbp5, have been described previously. The igfbp4 gene is expressed in the anterior part of the liver from stage 38 through 42 (Zhu et al., 2008). However, its detailed expres-

\footnotetext{
Abbreviations used in this paper: IGF, insulin-like growth factor; IGFBP, insulin-like
} growth factor binding protein.

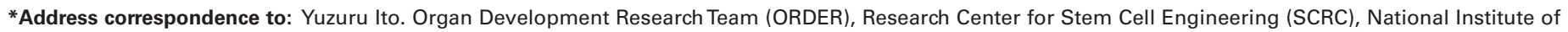

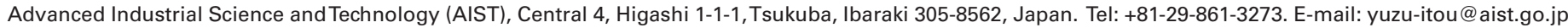

\#Present address: Department of Physiology, Kitasato University School of Medicine, 1-15-1 Kitasato, Minami, Sagamihara, Kanagawa 252-0374, Japan
} 
sion pattern has not been described. The expression of igfbp5 is detected maternally, and becomes localized to the floor plate, notochord, and dorsal endoderm during neurulation. At the tailbud stages, additional expression is detected in cranial nerves, ear vesicles, dorsal fins, and somites (Pera et al., 2001). X. tropicalis is a useful model animal for the study of early developmental functions of various genes. However, as mentioned above, the specific expression patterns of igfbpgenes during embryogenesis have not been completely described, and further data are needed. In this study, we showed that the $X$. tropicalis genome lacks the igfbp3 and igfbp6 genes. We detected four igfbp genes in $X$. tropicalis embryos, and documented their spatial and temporal expression patterns during early embryonic development.

\section{Result and Discussion}

\section{Cloning of four $\mathrm{X}$. tropicalis igfbp genes}

We identified four igfbp genes, igfbp1, igfbp2, igfbp4, and igfbp5 in $X$. tropicalis (Fig. 1A). In mice and humans, igfbp2 and igfbp5 are located on the same chromosome as a tandem repeat,
A
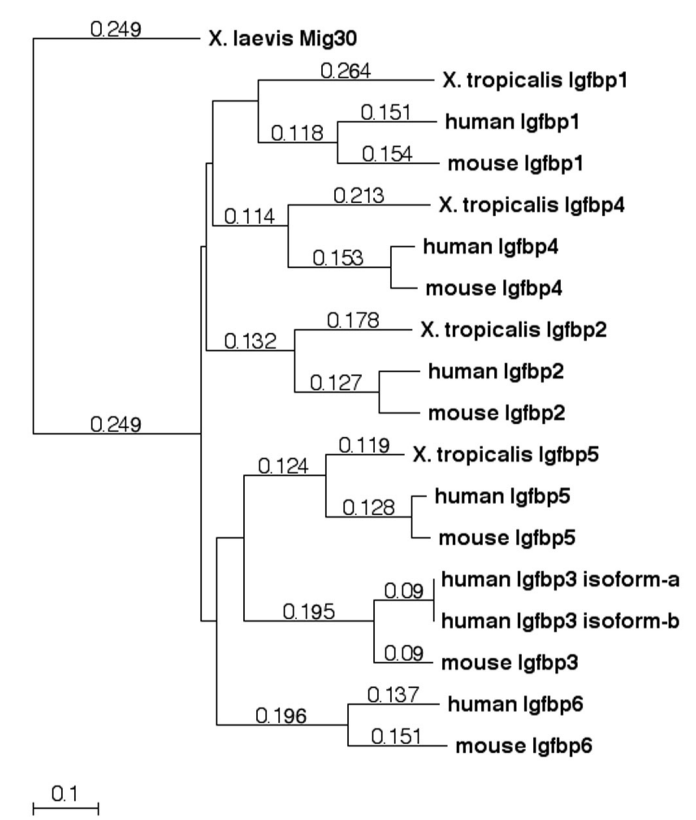

C

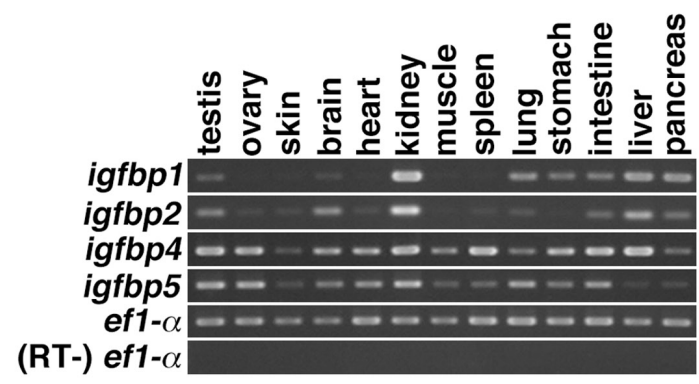

B
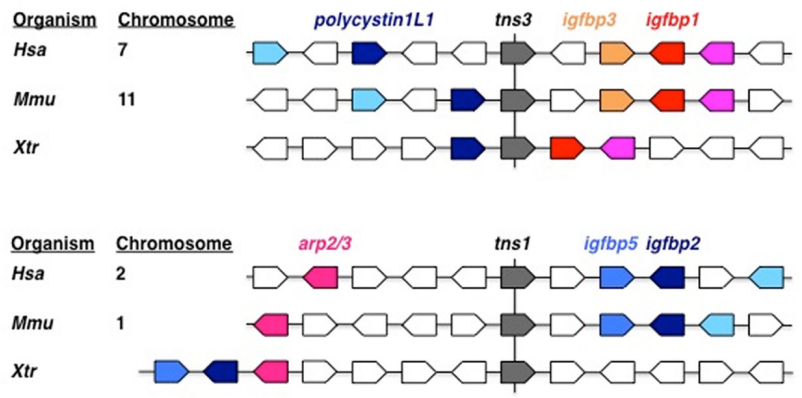

Organism Chromosome smarce1 ccr7 tns4 igfbp4 connexin31.9 Hsa $17 \quad \square-\mathrm{O}-\mathrm{O}-\mathrm{O}$ Mmu $11 \quad \square \square-\square \square$

$X t r$ DNA topoisomerace /l

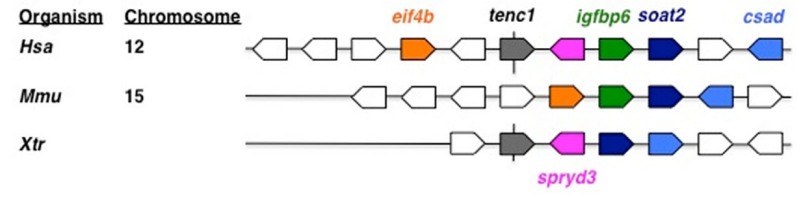

Hsa: H. sapiens Mmu: M. musculus

D

Fig. 1. Isolation of $\boldsymbol{X}$. tropicalis igfbp genes and their expression patterns in embryonic and adult tissues. (A) Phylogenetic tree of lgfbp amino acid sequences. The phylogenetic tree was calculated by MacVector 11.1.0 software. X. laevis Mig30 (GenBank accession No. NP_001082206) (Hayata et al., 2002 and Kuerner et al., 2006) was used as an outgroup. Sequence sources (i.e., GenBank Accession Nos.) are as follows: X. tropicalis Igfbp1 (NP_001029118), Igfbp2 (NP_001093707), Igfbp4 (XP_002942630), Igfbp5 (NP_001016042); Mus musculus lgfbp1 (NP_032367), lgfbp2 (NP_032368), Igfbp3 (NP_032369), Igfbp4 (NP_034647), Igfbp5 (NP_034648), Igfbp6 (NP_032370); Homo sapiens Igfbp1 (NP_000587), Igfbp2 (NP_000588), Igfbp3 isoform-a (NP_001013416), Igfbp3 isoform-b (NP_000589), Igfbp4 (NP_001543), Igfbp5 (NP_000590), and lgfbp6 (NP_002169). (B) Synteny analysis of igfbp genes. Synteny of igfbp genes is conserved among human, mouse, and X. tropicalis. Tensin genes near igfbp loci are shown in grey, and orthologs are connected with a vertical line. Orthologs are shown in the same colors, and genes whose positions are not conserved among human, mouse, and X. tropicalis are shown in white. In X. tropicalis, igfbp3 and igfbp6 seem to be lost. (C) The expression of igfbp1, 2, 4, and 5 in adult tissues of X. tropicalis. (D) Temporal expression of igfbp1, 2, 4, and 5 during early development of X. tropicalis. Numbers indicate developmental stages. 


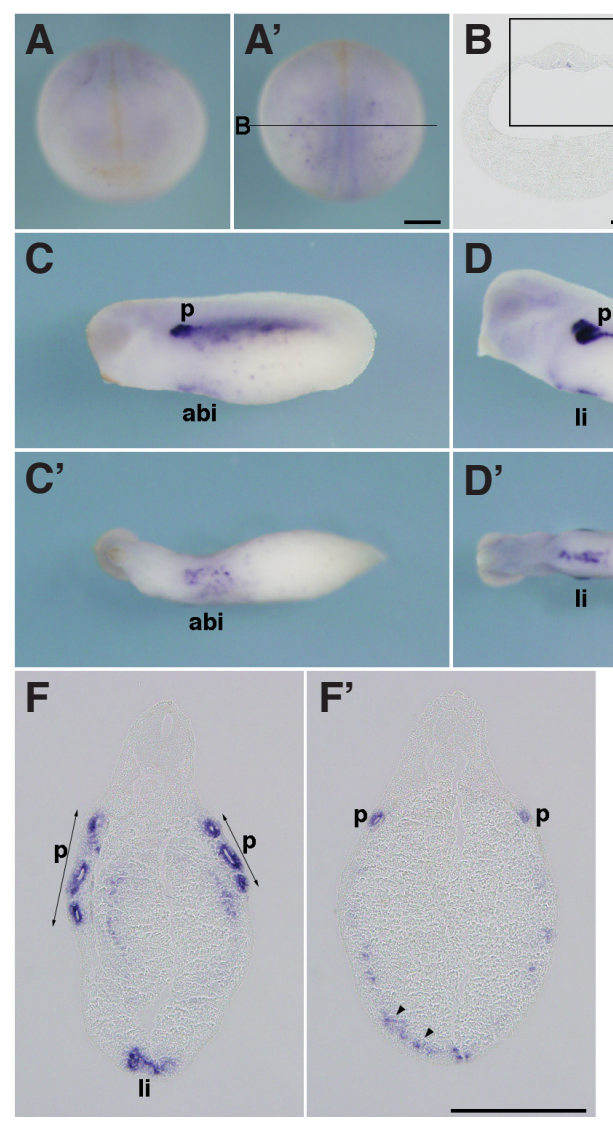

but are orientated in opposite transcription directions. The igfbp 1 and igfbp3 genes are linked in the same manner. The igfbp4 and igfbp6 genes are located on separate chromosomes (Fig. 1B). All igfbp genes are located near tensin-like genes (tns) in the mouse and human genomes. However, $X$. tropicalis igfbp3 and igfbp6 could not be identified in the proximity of ths loci (Fig. 1B). Furthermore, orthologous igfbp3 and igfbp6 sequences were not identified. Zebrafish and medaka genomes contain igfbp3 and igfbp 6 genes. Our synteny analysis showed that $X$. tropicalis lacks igfbp3and igfbp6 in the genome. Therefore, determining the function of each igfbp, and in particular, determining which Xenopus genes share the functions of igfbp3 and igfbp6 is intriguing.

\section{Expression of igfbp1}

Mouse igfbp1 expression has been detected in the liver after day 12 of gestation (Shuller et al., 1993a, b; Schuller et al., 1994). No expression was detected in other adult mouse tissues (Schuller et al., 1994). In humans, igfbp1 is most abundantly expressed in the fetal liver (Han et al., 1996). Our results indicated that $X$. tropicalis igfbp1 is expressed in various adult tissues, unlike in mouse and human. $X$. tropicalis igfbp1 was ex-

.

Fig. 2. In situ hybridization analysis of igfbp1 during $X$. tropicalis development. (A,A') Stage 20, (A) Anterior view (A') dorsal view. (B,B') Transversal section of an embryo at stage 20. (B') Magnified view of the boxed area in (B). Dorsal side is displayed towards the top. Igfbp1 is expressed in part of the archenteron roof near the dorsal midline. (C,C') Stage 25, (D,D') stage 30, (E,E') stage 35, (C,D,E) lateral view, $\left(\mathbf{C}^{\prime}, \mathbf{D}^{\prime}, \mathbf{E}^{\prime}\right)$ ventral view. $\left(\mathbf{F}, \mathbf{F}^{\prime}\right)$ Transversal section of an embryo at stage 35. Positions of sections are indicated by black lines, and letters mark corresponding panels. ar, archenteron roof; abi, anterior blood islands; li, liver; $p$, pronephric tubule. Arrowheads indicate scattered blood-like cells. Scale bars indicate $200 \mu \mathrm{m}$. pressed in the kidney, lung, stomach, intestine, liver, and, pancreas and was detected at low levels in the testis and brain (Fig. 1C). $X$. tropicalis igfbp1 was developmentally expressed from the late neurula stage (Fig. 1D, Fig. 2 A-F'). In the neurula (stage 20), igfbp1 was expressed in part of the archenteron roof near the dorsal midline (Fig. 2 A-B'). At the tailbud and tadpole stages (stage 25-35), igfbp1 was predominantly expressed in the pronephros (Fig. 2 C-F'). Igfbp1 was also expressed in anterior blood islands at stage 25 (Fig. $2 \mathrm{C}$ and $\mathrm{C}^{\prime}$ ), in a region around the liver at stage 30 (Fig. 2 D and D'), and in the liver and scattered blood-like cells at stage 35 (Fig. 2 E-F').

\section{Expression of igfbp2}

Expression of mouse igfbp2 has been detected in neural tissues as early as day 11 of gestation. The mouse igfbp2 transcript was detected in differentiating sclerotomes, the esophagus, nasal placode, lung, and liver starting on day 13. After day 14, the expression of mouse igfbp2 was also found in other tissues such as the eye, meninges, vertebrae, kidney, and intestine (Schul-
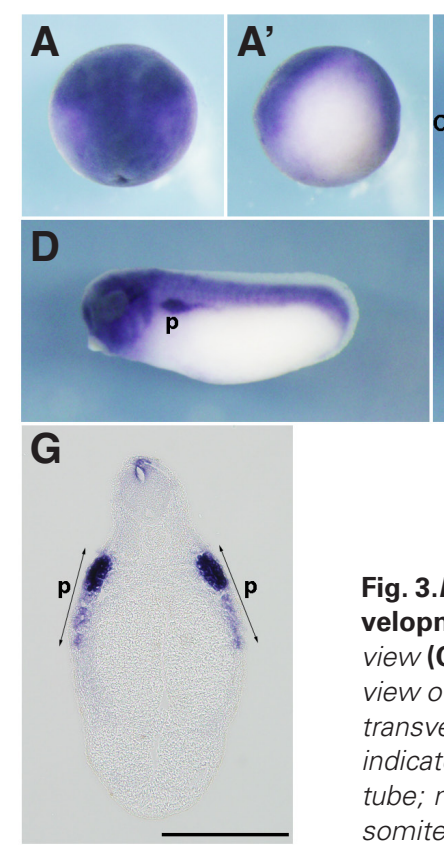

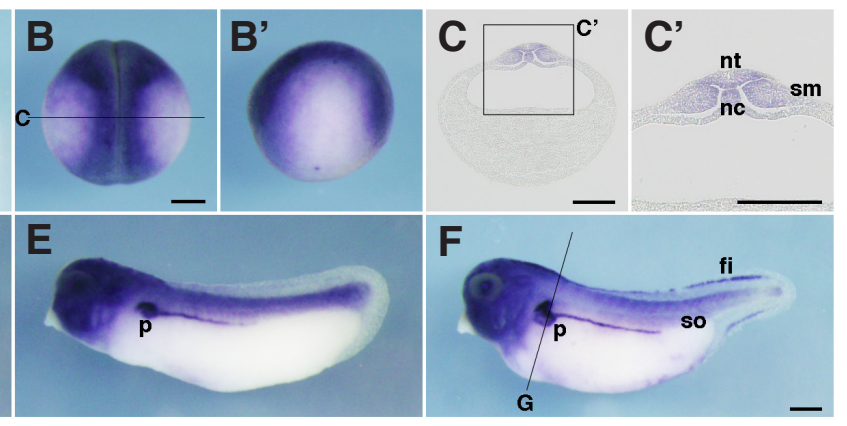

Fig. 3.In situ hybridization analysis of igfbp2 during $X$. tropicalis development. (A,A') Stage 15(B,B') stage 20 (A,B) dorsal view (A', $\left.\mathbf{B}^{\prime}\right)$ lateral view $\left(\mathbf{C}, \mathbf{C}^{\prime}\right)$ Transversal section of an embryo at stage 20. (C') Magnified view of the boxed area in (C). (D) Stage 25 (E) stage 30 (F) stage 35 (G) transversal section of an embryo at stage 35. Positions of sections are indicated by black lines, and letters mark corresponding panels. nt, neural tube; nc, notochord; sm, somatic mesoderm; p, pronephric tubule; so, somite; fi, fin. Scale bars indicate $200 \mu \mathrm{m}$. 


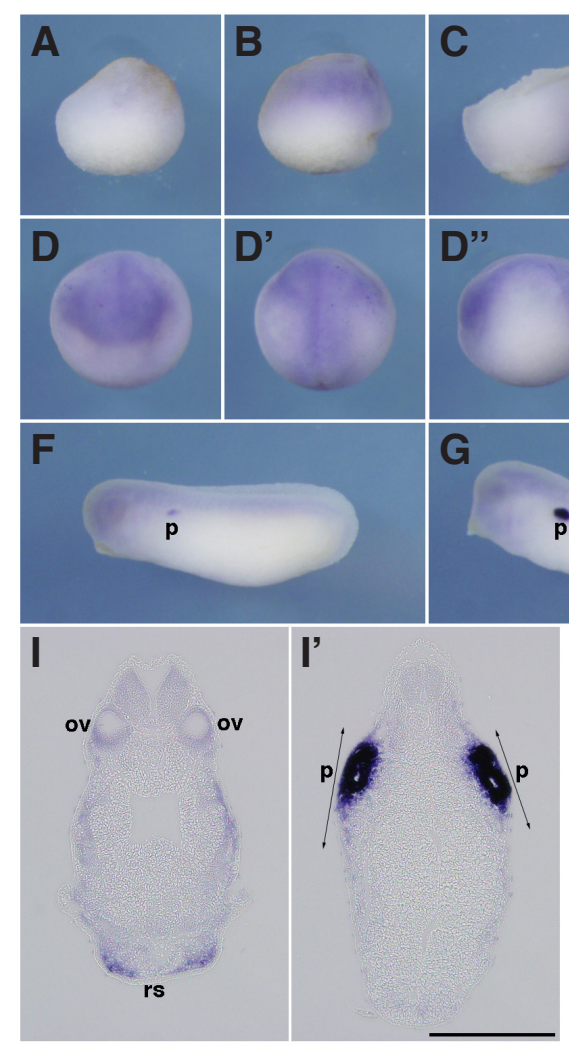

Fig. 4. In situ hybridization analysis of igfbp4 during $\boldsymbol{X}$. tropicalis development. (A) Stage 9, (B) stage 10.5, (C, $\mathbf{C}^{\prime}$ ) stage 12, (C) lateral view, (C') vegetal view, (D,D',D") stage 15, (E,E',E") stage 20, (D,E) anteriorview, (D',E') dorsal view, (D",E") lateral view, (F) stage 25, (G) stage $30, \mathbf{( H )}$ stage $35,\left(\mathbf{I}, \mathbf{I}^{\prime}\right)$ transversal section of an embryo at stage 35. Positions of sections are indicated by black lines, and letters mark corresponding panels. $p$, pronephric tubule; ov, otic vesicle; rs, rostal lymph sacs. Scale bars indicate $200 \mu \mathrm{m}$

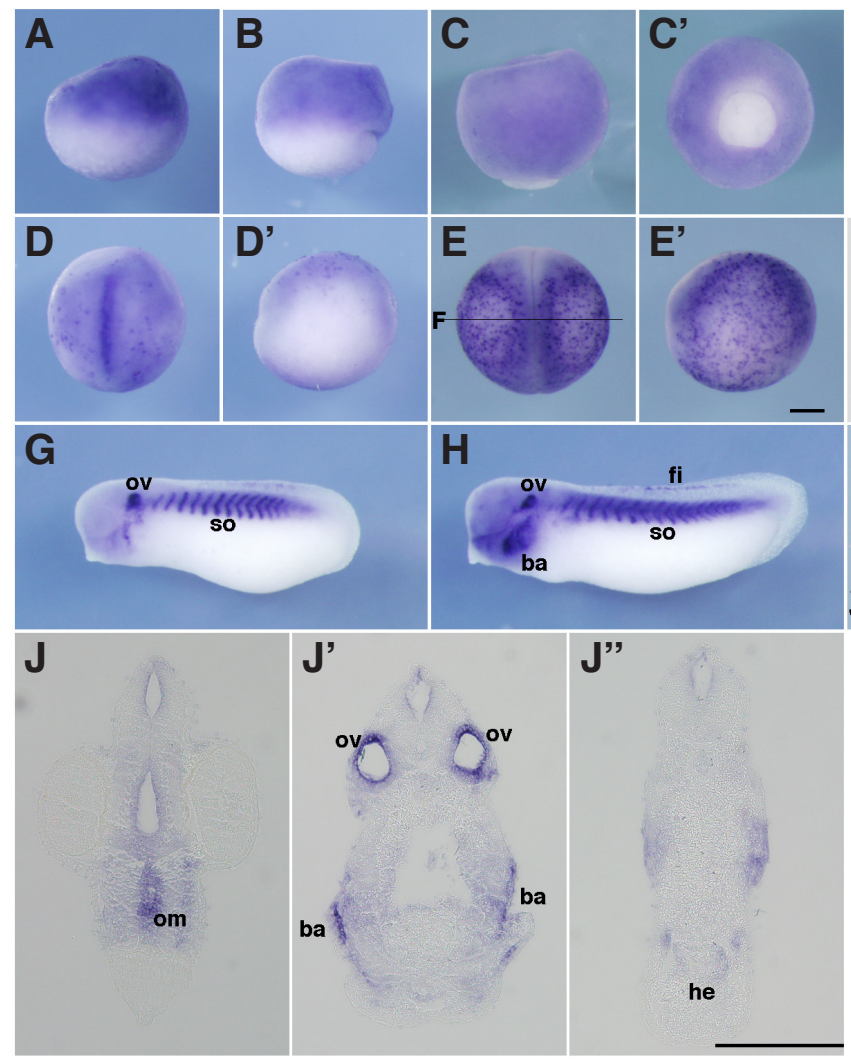

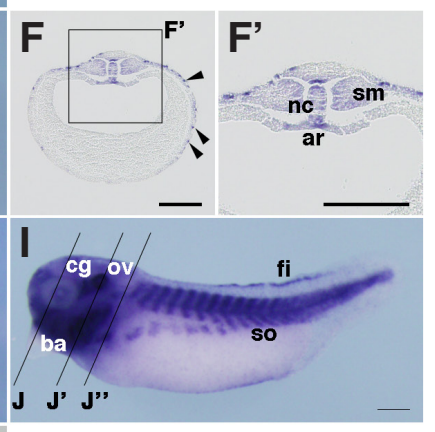

Fig. 5. In situ hybridization analysis of igfbp5 during $X$. tropicalis development. (A) Stage 9, (B) stage 10.5, (A,B) lateral view, (C,C') stage 12, (C) lateral view, (C') vegetal view, (D,D') stage 15, (E,E') stage 20, (D,E) dorsal view, (D',E') lateral view, (F,F') transversal section of an embryo at stage 20. $\left(\mathbf{F}^{\prime}\right)$ Magnified view of the boxed area in (F). (G) Stage 25, (H) stage 30, (I) stage $35,(\mathbf{J}, \mathbf{J}, \mathbf{J}$,$) transversal section of an embryo at stage 35. Positions of$ sections are indicated by black lines, and letters mark corresponding panels. nc, notochord; ar, archenteron roof; sm, somatic mesoderm; ov, otic vesicle; so, somite; ba, branchial arch; fi, fin; cg, cranial ganglia; om, oral membrane; ov, otic vesicle; he, heart. Scale bars indicate $200 \mu \mathrm{m}$. such as the lung, liver, kidney, intestine, and vertebrae (Schuller et al., 1993a, b). High expression of mouse igfbp 4 is detected in the adult liver, kidney, and spleen, and is also detectable in the lung, heart, spleen, and muscle. No heen detected in the testis or ovary (Schuller et al., 1994). at moderate levels in all tissues during gestational ages $10-16$ weeks (Han et ler et al., 1993a, b). High expression of mouse igfbp2 has been detected in the adult liver and kidney, and is also detectable in the lung, spleen, brain, testis, and ovary (Schuller et al., 1994). No expression was detected in muscle tissues. In humans, igfbp2 is expressed at moderate levels in every tissue, and is highest in the liver during gestational ages 10-16 weeks (Han et al., 1996). $X$. tropicalis igfbp2 was expressed in the testis, brain, kidney, intestine, liver, and pancreas, and was detected at low levels in the ovary, skin, heart, spleen, lung (Fig. 1C). The expression of X. tropicalis igfbp2 was developmentally Fig. 3A-G). At the neurula stage (stages 15-20), igfbp2 was broadly expressed around the dorsal region (Fig. $3 \mathrm{~A}-\mathrm{C}^{\prime}$ ), i.e., in the neural tube, somite, and notochord (Fig. 3 C and C'). At the tailbud and tadpole stages (stages 25-35), igfbp2 was predominantly expressed in the pronephros and neural region (Fig. 3D-G). At stage 35, igfbp2 was also detectable in part of the fin (Fig. 3F). There were no remarkable differences between the expression of igfbp2 in $X$.

\section{Expression of igfbp4}

Transcripts of mouse igfbp4 have been detected as early as day 11 in neural tissues and differentiating sclerotomes. After day 14, mouse igfbp 4 expression is decreased in the brain, and is clearly detected in other tissues 
al., 1996) and is most abundant in the kidney, stomach, intestine, and lung and least abundant in the liver during gestational ages 14-18 weeks (Delhanty et al., 1993). X. tropicalis igfbp4 was expressed in almost all tissues tested including the testis, ovary, brain, heart, kidney, muscle, spleen, lung, stomach, intestine, liver, and pancreas, and was detected at low levels in the skin (Fig. 1C). $X$. tropicalis igfbp4 expression increased gradually during development (Fig. 1D, Fig. 4 A-I'). At the blastula and gastrula stages (stages 9-12), igfbp4 was expressed in animal region, and gradually diminished thereafter (Fig. 4A-C'). At the neurula stage (stages 15-20), igfbp4 was expressed in the neural region (Fig. 4 D-E'). At the tailbud and tadpole stages (stages 25-35), igfbp 4 was predominantly expressed in the pronephros. At stage 35 , igfbp 4 was also detectable in the otic vesicle and rostral lymph sacs (Fig. $4 \mathrm{H}-\mathrm{I}$ '). X. tropicalis igfbp4 was expressed in the testis and ovary, unlike mouse igfbp 4 .

\section{Expression of igfbp5}

Expression of mouse igfbp5 is detectable as early as day 11 of gestation in differentiating sclerotomes. Expression of mouse igfbp5in 14-day-old embryos has been found in the nasal placodes, pharynx, and esophagus. After day 14 of gestation, expression of mouse igfbp5 has been found in tissues such as the cornea and sclera of the eye, meninges, lung, kidney, intestine, and vertebrae (Schuller et al., 1993a, b). High expression of mouse igfbp5 is detected in the adult kidney, muscle, and ovary, and is also detectable in the lung, heart, brain, and testis (Schuller et al., 1994). In humans, igfbp5 is expressed most abundantly in the skin, muscle, and stomach during gestational ages 10-16 weeks (Han et al., 1996) and is most abundant in the muscle, skin, stomach, and intestine during gestational ages 14-18 weeks (Delhanty et al., 1993). X. tropicalis igfbp5 was expressed in almost all tissues tested, including the testis, ovary, brain, heart, kidney, muscle, spleen, lung, stomach, and intestine, and was detected at low levels in the skin, liver, and pancreas (Fig. 1C). X. tropicalis igfbp5 was maternally expressed. The expression of igfbp5 gradually decreased until the neurula stage (stage 15), and then increased from the late neurula stage (stage 20) (Fig. 1D, Fig. 5A-J"). At the blastula and gastrula stages (stages 9-12), igfbp5 was broadly expressed in the animal hemisphere (Fig. 5 A-C'). At the neurula stage (stages 15-20), igfbp5 was expressed in the notochord, somite, the dorsal part of the archenteron roof, and in scattered cells in the ectodermal epithelium (Fig. 5 D-F'). At the tailbud and tadpole stages (stages 25-35), igfbp5 was predominantly expressed in the somite, otic vesicle, branchial arch, and part of the fin (Fig. 5 G-J"). At stage 35, igfbp5 was also detectable in the oral membrane, cranial ganglia, and heart (Fig. 5 I-J').

\section{Expression of igfbps in the pronephric kidney}

In this study, we focused on the expression of igfbps in the pronephros, because all igfbp genes except for igfbp5 were expressed in the pronephric tubule or duct. Synteny analysis indicated that the $X$. tropicalis genome lacks igfbp3 and igfbp6. Therefore, functional validation of the lack of these genes in $X$. tropicalis development is of interest. High expression of mouse igfbp3 has been detected in the adult kidney, and is detectable in the liver, lung, heart, spleen, and muscle (Schuller et al., 1994). Importantly, igfbp1, which is not detected in the kidney in the mouse or human, was highly expressed in the pronephric tubule and duct and the adult kidney of $X$. tropicalis. This expression of igfbp 1 might compensate for the lack of igfbp3, which is nearby in the mice and human genomes. High expression of $X$. tropicalis igfbp 1 was detected in region 3 of the proximal tubule (PT), all regions of the intermediate tubule (IT), and the connecting tubule (CT), and was detectable in all regions of the distal tubule (DT) (Fig. 6 A-B' and G). Igfbp1 was also expressed in the nephrostome, and probably in PT1 (Fig. 6 A-A' and G). High expression of $X$. tropicalis igfbp2 was detected in PT1-3, DT1-2, and CT, and was
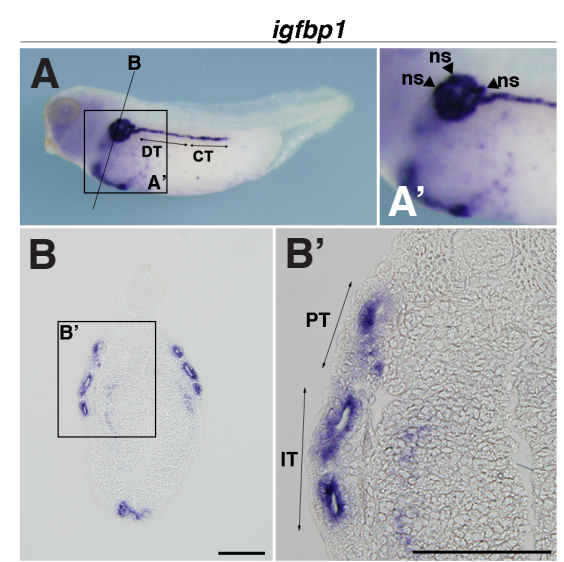

G
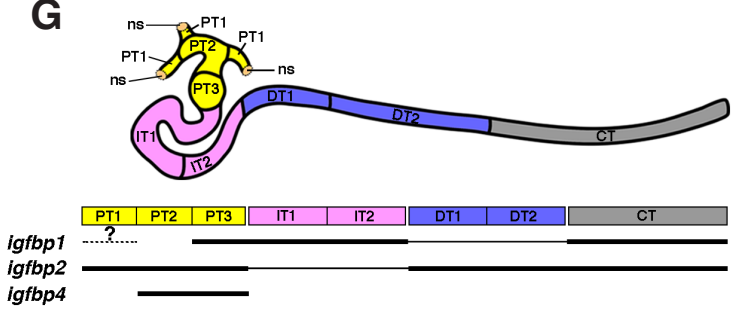

igfbp2
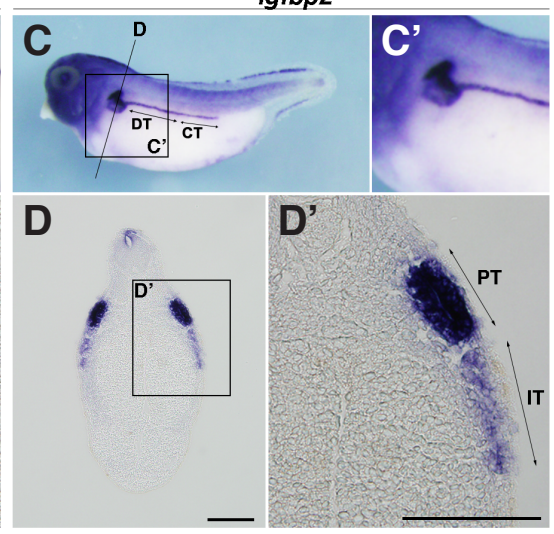

Fig. 6. Comparative expression patterns of igfbps in pronephros development. (A-B') igfbp1 (C-D') igfbp2 (E-F') igfbp4 expressions at stage $35\left(\mathbf{A}^{\prime}, \mathbf{B}^{\prime}, \mathbf{C}^{\prime}, \mathbf{D}^{\prime}, \mathbf{E}^{\prime}, \mathbf{F}^{\prime}\right)$ magnified view of the boxed area in $(\mathbf{A}, \mathbf{B}, \mathbf{C}, \mathbf{D}, \mathbf{E}, \mathbf{F})$, respectively. $\left(\mathbf{B}, \mathbf{B}, \mathbf{D}, \mathbf{D}, \mathbf{F}, \mathbf{F}^{\prime}\right)$ Transversal sections. Positions of sections are indicated by black lines, and letters mark corresponding panels. (G) Summary of igfbp expression in pronephros. This is a modified illustration. The original schematic diagram was described in Raciti et al., (2008). The thickness of lines represents the intensity of staining by whole-mount in situ hybridization. ns, nephrostome; PT, proximal tubule; IT, intermediate tubule; DT, distal tubule; CT, connecting tubule; ns, nephrostome. Scale bars indicate $200 \mu \mathrm{m}$. 
TABLE 1

\section{RT-PCR PRIMER SEQUENCES}

\begin{tabular}{|c|c|c|c|c|c|c|}
\hline Gene & Primer sequence & Annealing temperature $\left({ }^{\circ} \mathrm{C}\right)$ & Cycles & Length (bp) & Accession no. & Reference \\
\hline igfbp1 & $\begin{array}{l}\text { 5'-GCTGCCTGACTTGTGCTCTAAAG-3', } \\
\text { 5'-CGAAGAAATGGTGGAATCTGGTC-3' }\end{array}$ & 55 & 35 & 265 & BC099978 & new \\
\hline igfbp2 & $\begin{array}{l}\text { 5'-ATGAGCAGCAGCGGTCAAAG-3' } \\
\text { 5'-TTCACACACCAGCATTCCCC-3' }\end{array}$ & 55 & 35 & 244 & BC135928 & new \\
\hline igfbp4 & $\begin{array}{l}\text { 5'-CCCTGCCCTTTTGGTTGCTTG-3' } \\
\text { 5'-GGACTCAATCTCCCCAATCTCAG-3' }\end{array}$ & 55 & 35 & 301 & XM_002942584 & new \\
\hline igfbp5 & $\begin{array}{l}\text { 5'-TGTGAGCCCTGCGATGATAAAG-3' } \\
\text { 5'-TTCTGAGGTGGTCGGTTCTTCG-3' }\end{array}$ & 55 & 35 & 288 & CR848090 & new \\
\hline odc & $\begin{array}{l}\text { 5'-GCACATGTCAAGCCAGTTCT-3' } \\
\text { 5'-TGCGCTCAGTTCTGGTACTT-3' }\end{array}$ & 60 & 22 & 303 & NM_001005441 & Haramoto et al., 2004 \\
\hline ef1a & $\begin{array}{l}\text { 5'-TGTAGGAGTCATCAAGGCGGTC-3' } \\
\text { 5'-ACAGATTTTGGTCAAGTTGCTTCC-3' }\end{array}$ & 60 & 22 & 321 & NM_001016692 & Fukuda et al., 2010 \\
\hline
\end{tabular}

detectable in DT1-2 (Fig. 6 C-D' and G). High expression of $X$. tropicalis igfbp4 was detected only in PT2-3 (Fig. 6 E-F' and G). Our results showed that at least two igfbps were co-expressed in all pronephric domains, suggesting that redundant function of igfbp genes is required in early pronephric kidney development.

In conclusion, we identified $X$. tropicalis igfbp genes and examined their spatial and temporal expression patterns during early embryonic development. Such distinct expression patterns of igfbp genes suggest divergent roles in embryonic development. Redundancy and tandem repeats in the genome make it difficult to understand their in vivo function. The genome of $X$. tropicalis has fewer igfbp genes than mammalian genomes. This small number of igfbp genes indicates that $X$. tropicalis is suitable for loss of function analysis of the Igfbp family. Our study will facilitate functional analysis of the Igfbp family during embryonic development.

\section{Materials and Methods}

\section{Genome Analysis}

Using Metazome v3.0 (http://www.metazome.net/) and the Xenbase genome browser ( $X$. tropicalis-ver. 7.1) (http://gbrowse.xenbase.org/ fgb2/gbrowse/xt7_1/?), the upstream and downstream flanking genes of igfbp orthologs were compared between $H$. sapiens, M. musculus, and $X$. tropicalis. The neighbor joining phylogenetic tree was calculated using MacVector 11.1 .0 software.

\section{Cloning of X. tropicalis igfbp genes}

$X$. tropicalis igfbp sequences were RT-PCR amplified from total cDNA using gene-specific primers. Gene-specific primers were as follows: igfbp1 (NM_001033946) (F, 5'-CACACTCGAGATGGCTAGGGAGAACATCTC-3'; R, 5'-CACATCTAGACTATTCTTGAACATTAAGGTAC-3'), igfbp2 (NM_001100237) (F, 5'-CACAGAATTCATGGGGCTCAGCCGGTACCTG-3'; R, 5'-CACATCTAGACTACGGGGCCCGCTGAGTATG-3'), igfbp4 (XM_002942584) (F: 5'-CACAGAATTCATGTCTGGAAACTGCCACCC-3'; R, 5'-CACACTCGAGTCATTCCTTTCCCCTCTCAG-3'), and igfbp5 (NM_001016042) (F, 5'-ATGGAAATGTTGGTGCCAGC-3'; igfbp5 R, $5^{\prime}$-TCATTCTGTGTTGCTGCTATC-3'). The PCR products were digested with Xhol/Xbal for igfbp1, EcoRl/Xbal for igfbp2, EcoRl/Xhol for igfbp4 and cloned into the corresponding site of the pCS2p vector. The PCR product for igfbp5 was cloned into the Stul site of the pCS2p vector.

\section{$R T-P C R$}

Total RNA was extracted from $X$. tropicalis embryos and adult tissues (Nigerian line) using ISOGEN (Nippon Gene, Toyama, Japan) by homogenization (Physcotron, Microtec Co., Ltd., Chiba, Japan). First-strand
cDNA was synthesized from $1 \mu \mathrm{g}$ of total RNA using Superscript III reverse transcriptase (Life Technologies, Carlsbad, CA, USA). Ornithine decarboxylase $(\mathrm{odc})$ and elongation factor $1 \alpha(e f 1 \alpha)$ were used as internal controls. Reverse transcriptase negative (RT-) reactions indicated the absence of genomic DNA contamination. Primer sequences, sizes of PCR products, and cycling numbers are described in Table 1.

\section{Embryos and whole-mount in situ hybridization}

$X$. tropicalis embryos were obtained by artificial fertilization, and cultured in $0.1 \times$ Steinberg's solution (Haramoto et al., 2004). The embryos were staged according to Nieuwkoop and Faber (1956). Whole-mount in situ hybridization was carried out as previously described (Sive et al., 2000). DIG-labeled antisense RNA probes were synthesized with T7 polymerase (Promega, Madison, WI, USA) using the following plasmids: pCS2p-igfbp1, pCS2p-igfbp2, pCS2p-igfbp4, and pCS2p-igfbp5. After whole-mount in situ hybridization, embryos were embedded in paraffin, sectioned into thin slices $(10 \mu \mathrm{m})$, and observed under an optical microscope (BX51; Olympus, Tokyo, Japan).

\section{Acknowledgments}

$X$. tropicalis was provided by the National Bio-Resource Project (NBRP) of the MEXT, Japan. This study was supported by JSPS KAKENHI Grant Number 24590232, 21241048. The authors report no conflicts of interest.

\section{Author contributions}

Conceived and designed the experiments: YH, YI. Performed the experiments: $Y H, T O$, and ST. Wrote the paper: $Y H$.

\section{References}

DELHANTY, PJD, HILL, DJ, SHIMASAKI, S, HAN, VKM (1993). Insulin-like growth factor binding protein-4, -5 and- 6 mRNAs in the human fetus: Localization to sites of growth and differentiation? Growth Regulation 3: 8-11.

FUKUDA, M, TAKAHASHI, S, HARAMOTO, Y, ONUMA, Y, KIM, YJ, YEO, CY, ISHIURA, S, ASASHIMA, M (2010). Zygotic VegT is required for Xenopus paraxial mesoderm formation and is regulated by Nodal signaling and Eomesodermin. Int. J. Dev. Biol. 54: 81-92.

HAN, VKM, MATSELL, DG, DELHANTY, PJD, HILL, DJ, SHIMASAKI, S, NYGARD, K (1996). IGF-binding protein mRNAs in the human fetus: tissue and cellular distribution of developmental expression. Horm. Res. 45: 160-166.

HARAMOTO, Y, TANEGASHIMA, K, ONUMA, Y, TAKAHASHI, S, SEKIZAKI, H, ASASHIMA, M (2004). Xenopus tropicalis nodal-related gene 3 regulates BMP signaling: an essential role for the pro-region. Dev Biol. 265: 155-168.

HAYATA, T, TANEGASHIMA, K, TAKAHASHI, S, SOGAME, A, ASASHIMA, M (2002) Overexpression of the secreted factor Mig30 expressed in the Spemann organizer impairs morphogenetic movements during Xenopus gastrulation. Mech. Dev. 112: 37-51.

HWA, V, OH, Y, ROSENFELD, RG (1999). The insulin-like growth factor binding 
protein (IGFBP) superfamily. Endocrine Rev. 20: 761-787.

KUERNER, KM, STEINBEISSER, H (2006). Expression analysis of IGFBP-r10, IGFBP-like and Mig30 in early Xenopus development. Dev. Dyn. 235: 2861-2867.

NAKAE, J, KIDO, Y, ACCILI, D (2001) Distinct and overlapping functions of insulin and IGF-I receptors. Endocr Rev. 22: 818-835.

NIEUWKOOP, PD, FABER, J (1956). Normal table of Xenopus laevis (Daudin); a systematical and chronological survey of the development from the fertilized egg till the end of metamorphosis, North-Holland Pub. Co., Amsterdam.

PERA, EM, WESSELY, O, LI, SY, DE ROBERTIS, EM (2001). Neural and head induction by insulin-like growth factor signals. Dev. Cell 1: 655-665

RACITI, D., REGGIANI, L., GEFFERS, L., JIANG, Q., BACCHION, F., SUBRIZI, A.E., CLEMENTS, D, TINDAL, C, DAVIDSON, DR, KAISSLING, B, BRÄNDL AW (2008). Organization of the pronephric kidney revealed by large-scale gene expression mapping. Genome Biol. 9: R84.

SCHULLER, AGP, GROFFEN, C, VAN NECK, JW, ZWARTHOFF, EC DROP, SLS
(1994). cDNA cloning and mRNA expression of the six mouse insulin-like growth factor binding proteins. Mol. Cell. Endocrinol. 104: 57-66.

SCHULLER, AGP, VAN NECK JW, LINDENBERGH-KORTLEVE, DJ, GROFFEN, C, DE JONG, I, ZWARTHOFF, EC, DROP, SL (1993). Gene expression of the IGF binding proteins during post-implantation embryogenesis of the mouse comparison with the expression of IGF-I and -II and their receptors in rodent and human. Adv. Exp. Med. Biol. 343: 267-277.

SCHULLER, AGP, ZWARTHOFF, EC, DROP, SLS (1993). Gene expression of the six insulin-like growth factor binding proteins in the mouse conceptus during mid-and late gestation. Endocrinol. 132: 2544-2550.

SIVE, HL, GRAINGER, RM, HARLAND, RM (2000). Early Development of Xenopus laevis: A Laboratory Manual. Cold Spring Harbor Laboratory Press, New York.

ZHU, W, SHIOJIMA, I, ITO, Y, LI, Z, IKEDA, H, YOSHIDA, M, NAITO, AT, NISHI, J, UENO, H, UMEZAWA, A, MINAMINO, T, NAGAI, T, KIKUCHI, A, ASASHIMA, M, KOMURO, I (2008) IGFBP-4 is an inhibitor of canonical Wnt signaling required for cardiogenesis. Nature 454: 345-350. 


\section{Further Related Reading, published previously in the Int. J. Dev. Biol.}

Evolving tales of autophagy in early reproductive events

Hyunjung J. Lim and Haengseok Song

Int. J. Dev. Biol. (2014) 58: 183-187

http://dx.doi.org/10.1387/ijdb.130337hl

Multiple DIk1 splice variants are expressed during early mouse embryogenesis

Antony J. Miller and Susan E. Cole

Int. J. Dev. Biol. (2014) 58: 65-70

http://dx.doi.org/10.1387/ijdb.130316sc

Hippo signaling components, Mst1 and Mst2, act as a switch between self-renewal and differentiation in Xenopus hematopoietic and endothelial progenitors

Susumu Nejigane, Shuji Takahashi, Yoshikazu Haramoto, Tatsuo Michiue and Makoto Asashima

Int. J. Dev. Biol. (2013) 57: 407-414

http://dx.doi.org/10.1387/ijdb.130010st

Induction of differentiation of undifferentiated cells into pancreatic beta cells in vertebrates

Masaki Hosoya, Yuya Kunisada, Akira Kurisaki and Makoto Asashima

Int. J. Dev. Biol. (2012) 56: 313-323

http://dx.doi.org/10.1387/ijdb.123522mh

Insulin-like growth factor binding proteins and mammary gland development Angara Sureshbabu, Elizabeth Tonner and David J. Flint

Int. J. Dev. Biol. (2011) 55: 781-789

http://dx.doi.org/10.1387/ijdb.113364as

Hematopoietic stem cell emergence in the conceptus and the role of Runx1 Gemma Swiers, Marella de Bruijn and Nancy A. Speck

Int. J. Dev. Biol. (2010) 54: 1151-1163

http://dx.doi.org/10.1387/ijdb.103106gs

Creating frog heart as an organ: in vitro-induced heart functions as a circulatory organ in vivo

Masayoshi Kinoshita, Takashi Ariizumi, Shinsuke Yuasa, Shunichirou Miyoshi, Shinji Komazaki, Keiichi Fukuda and Makoto Asashima

Int. J. Dev. Biol. (2010) 54: 851-856

http://dx.doi.org/10.1387/ijdb.093036mk

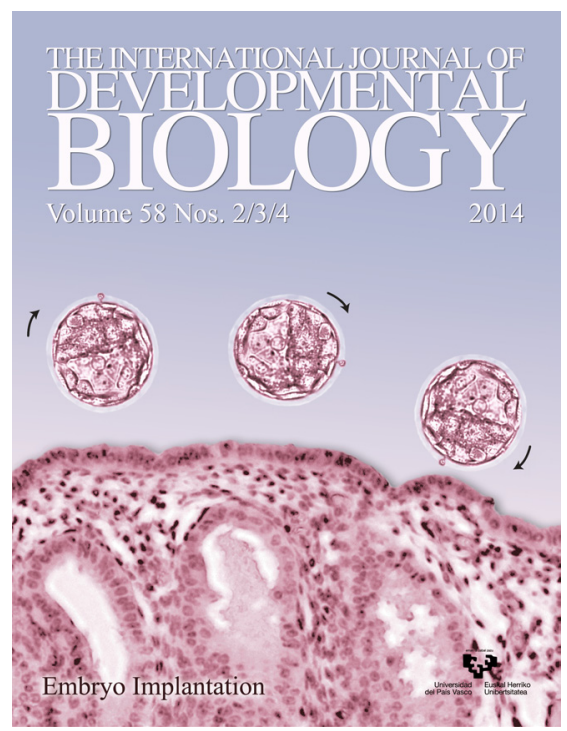

5 yr ISI Impact Factor $(2013)=2.857$
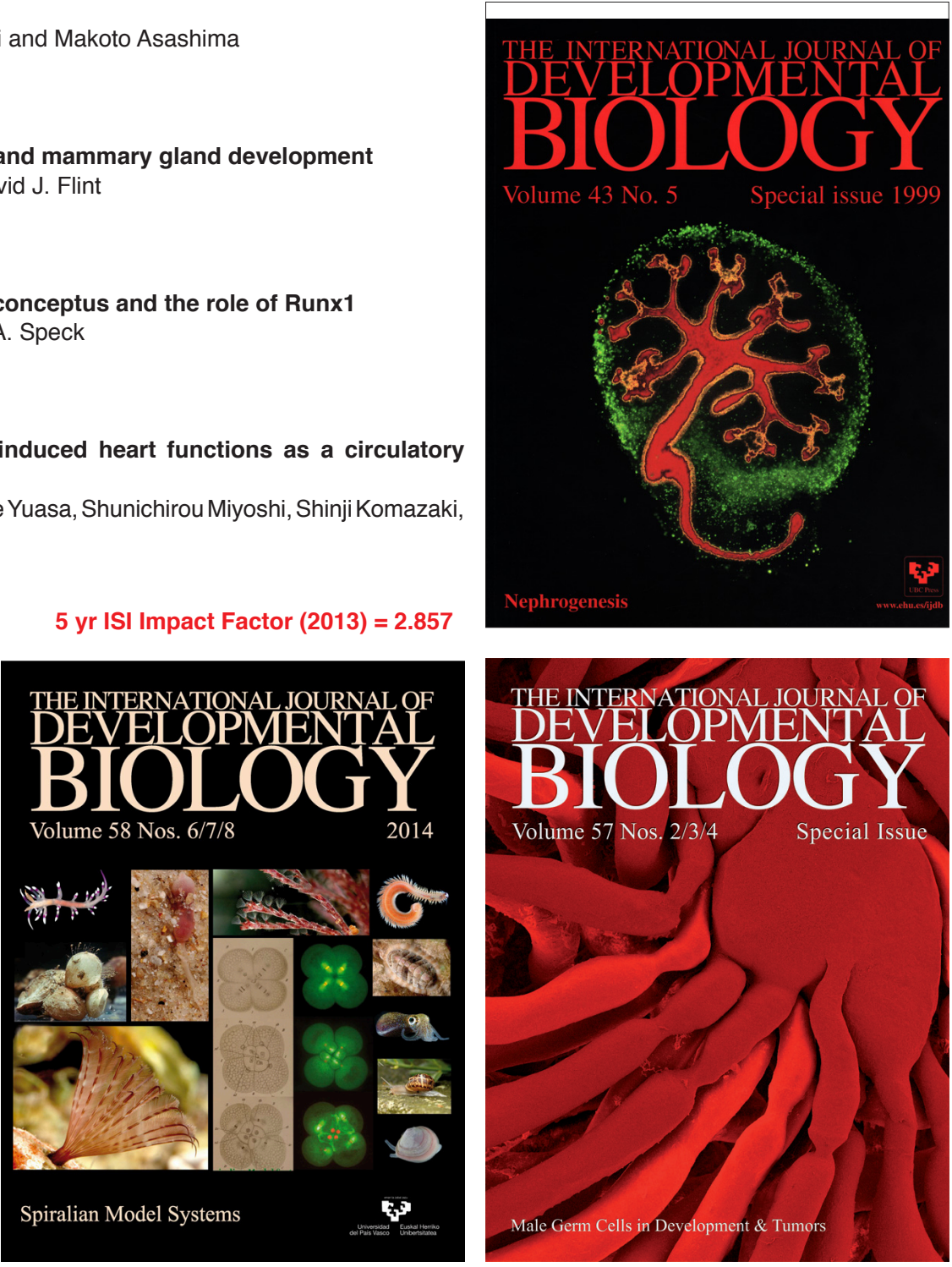

Spiralian Model Systems

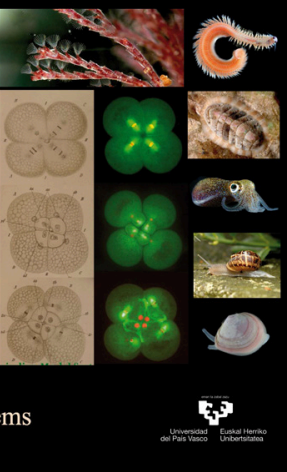

